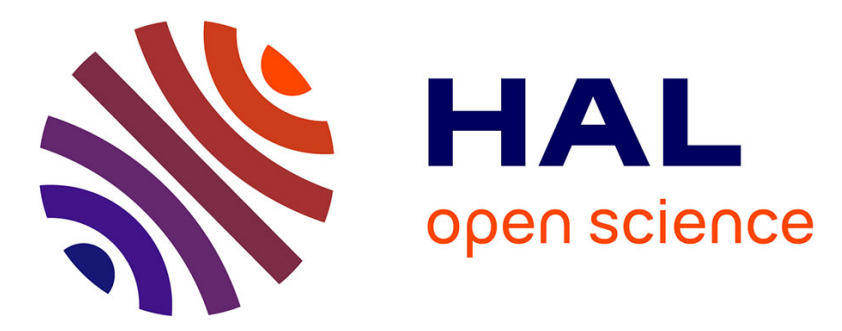

\title{
On the Geometry and the Deformation of Shapes Represented by Piecewise Continuous Bézier Curves with Application to Shape Optimization
}

\author{
Olivier Ruatta
}

\section{- To cite this version:}

Olivier Ruatta. On the Geometry and the Deformation of Shapes Represented by Piecewise Continuous Bézier Curves with Application to Shape Optimization. Geometric Science of Information, Aug 2013, Paris, France. pp.112-119, 10.1007/978-3-642-40020-9 . hal-00908903

\section{HAL Id: hal-00908903 https://hal.science/hal-00908903}

Submitted on 25 Nov 2013

HAL is a multi-disciplinary open access archive for the deposit and dissemination of scientific research documents, whether they are published or not. The documents may come from teaching and research institutions in France or abroad, or from public or private research centers.
L'archive ouverte pluridisciplinaire HAL, est destinée au dépôt et à la diffusion de documents scientifiques de niveau recherche, publiés ou non, émanant des établissements d'enseignement et de recherche français ou étrangers, des laboratoires publics ou privés. 


\title{
On the geometry and the deformation of shapes represented by piecewise continuous Bézier curves with application to shape optimization
}

\author{
Olivier Ruatta \\ XLIM - DMI UMR 7252 Université de Limoges - CNRS \\ olivier.ruatta@math.cnrs.fr
}

\begin{abstract}
In this work, we develop a framework based on piecewise Bézier curves to plane shapes deformation and we apply it to shape optimization problems. We describe a general setting and some general result to reduce the study of a shape optimization problem to a finite dimensional problem of integration of a special type of vector field. We show a practical problem where this approach leads to efficient algorithms.
\end{abstract}

In all the text below, $E=\mathbb{R}^{2}$. In this text, we will define a set of manifolds, each point of such a manifold is a parametrized curves in $E$.

\section{Bézier curves}

Bézier curves are usual objects in Computer Aided Geometric Design (CAGD) and have natural and straightforward generalization for surfaces and higher dimension geometrical objects. We focus here on plane curves even if most of results of this paper have a natural generalization in higher dimension. We will show that a Bézier curve is fully encoded by a set of points. This set of points forms the control polygon. This will lead us to a parametrization of curves by their control polygon giving to the set of Bézier (and piecewise Bézier) curves a manifold structure by diffeomorphism. This diffeomorphism is in fact linear and allows to studied space of curve as a vector space. Using this will be able to interpret deformations as infinitesimal curves of the same kind. The aim of this section is to fix notation and make the paper as self contained as possible.

\subsection{Basic definitions}

Given $P_{0}, P_{1}, \ldots, P_{D} \in E$, we define:

$$
B\left(\left(P_{0}, \ldots, P_{D}\right), t\right)=(1-t) B\left(\left(P_{0}, \ldots, P_{D-1}\right), t\right)+t B\left(\left(P_{1}, \ldots, P_{D}\right), t\right)
$$

with $B((P), t)=P$ for every $P \in E$. The associated Bézier curve is $\left\{B\left(\left(P_{0}, \ldots, P_{D}\right), t\right) \mid t \in[0,1]\right\}$ and the list $\left(P_{0}, \ldots, P_{D}\right)$ is called the control polygon and the points $P_{0}, \ldots, P_{D}$ are called the control points.

This process associates to every set of points a parametrized curve. It is a polynomially parametrized curve and its degree is bounded : 
Proposition 1. Let $P_{0}, \ldots, P_{D} \in E$, then $B\left(\left(P_{0}, \ldots, P_{D}\right), t\right)$ is a polynomial parametrization and its coordinates have degree at most $D$.

\subsection{Bernstein's polynomials}

Definition 1. Let $D$ be an integer and $i \in\{0, \ldots, D\}$, we define the Bernstein polynomial $b_{i, D}(t):=\left(\begin{array}{l}i \\ D\end{array}\right)(1-t)^{D-i} t^{i}$.

Notation 1 We denote $\mathbb{R}[t]_{D}$ the set of polynomial of degree less or equal to $D$. The set $\mathbb{R}[t]_{D}$ has a natural $\mathbb{R}$-vector space structure, its dimension is $D+1$ and $\left\{1, t, \ldots, t^{D}\right\}$ is a basis of this vector space.

Proposition 2. The set $\left\{b_{0, D}, \ldots, b_{D, D}\right\}$ is a basis of $\mathbb{R}[t]_{D}$.

Proposition 3. Let $P_{0}, \ldots, P_{D} \in E$, then $B\left(\left(P_{0}, \ldots, P_{D}\right), t\right)=\sum_{i=0}^{N} P_{i} b_{i, D}(t)$ for all $t \in[0,1]$.

Corollary 1. Every polynomially parametrized curve can be represented as a Bézier curve.

\subsection{Interpolation}

Since a Bézier curve of degree $D$ is defined using $D+1$ control points, one can hope to recover $D+1$ control points of a curve given a sampling of $D+1$ points on a curve. The following result shows that this is possible. But in fact, we do not have a single Bézier curve of degree $D$ but many. Each such curve is associated with a particular sampling of the parameter interval $[0,1]$.

Proposition 4. Let $M_{0}, \ldots, M_{D} \in E$, then there exists Bézier curves of degree $D$ passing through these points.

Lemma 1. Let $t_{0}=0<t_{1}<\cdots<t_{D}=1$, then there exists one and only one Bézier curve $B\left(\left(P_{0}, \ldots, P_{D}\right), t\right)$ of degree $D$ such that $B\left(\left(P_{0}, \ldots, P_{D}\right), t_{i}\right)=$ $M_{i}, \forall i \in\{0, \ldots, D\}$.

Proof. Denote by $M$ the $2 \times(D+1)$ matrix built with the coordinates of $M_{i}$ as $i^{\text {th }}$ row, i.e. $M=\left(M_{0}, \ldots, M_{D}\right)^{t}$, and denote by $P$ the $2 \times(D+1)$ matrix built with the coordinates of $P_{i}$ as $i^{\text {th }}$ row, i.e. $P=\left(P_{0}, \ldots, P_{D}\right)^{t}$. We consider the following matrix associated with $\boldsymbol{t}=\left(t_{0}, \ldots, t_{D}\right)$ :

$$
B_{\boldsymbol{t}, D}=\left(\begin{array}{cccc}
b_{0, D}(0) & b_{1, D}(0) & \cdots & b_{D, D}(0) \\
b_{0, D}\left(t_{1}\right) & b_{1, D}\left(t_{1}\right) & \cdots & b_{D, D}\left(t_{1}\right) \\
\vdots & \vdots & \ddots & \vdots \\
b_{0, D}(1) & b_{1, D}(1) & \cdots & b_{D, D}(1)
\end{array}\right)
$$

The matrix of equation (1) is invertible (it is the Vandermonde matrix expressed in the Bernstein basis) and clearly if $P$ is such that $B_{\boldsymbol{t}, D} P=M$, then $B\left(\left[P_{0}, \ldots, P_{D}\right], t\right)$ gives the wanted curve for the proof of the lemma. 
Remark that once $\boldsymbol{t}$ is known, one can compute $B_{\boldsymbol{t}, D}^{-1}$ once and for all, and that it is possible to take advantage of its Vandermonde-like structure in order to improve the cost of the multiplication of a vector by $B_{\boldsymbol{t}, D}$. Generally, we use a regular subdivision $\left(t_{i}=\frac{i}{D}\right)$ but there are more suitable choices in regard to the stability of the computation.

\section{Piecewize Bézier curves}

\subsection{Basics on piecewise Bézier curves}

Let $P_{0,0}, \ldots, P_{0, D}, P_{1,0}, \ldots, P_{1, D}, \ldots, P_{N, 0}, \ldots, P_{N, D} \in E$, such that $P_{i, D}=$ $P_{i+1,0}$ for all $i \in\{0, \ldots, N-1\}$, and let $\boldsymbol{P}_{i}=\left(P_{i, 0}, \ldots, P_{i, D}\right)$, we define:

$$
\Gamma\left(\boldsymbol{P}_{0}, \ldots, \boldsymbol{P}_{N}, t\right)=B\left(\boldsymbol{P}_{i}, \frac{i}{l+1}+(l+1) t\right)
$$

for $l \in\{0, \ldots, N\}, t \in\left[\frac{i}{(l+1)}, \frac{(i+1)}{(l+1)}\right]$ and for all $i \in\{0, \ldots, l\}$. This defines a continuous parametrization. The curves parametrized by $B\left(\left(P_{i, 0}, \ldots, P_{i, D_{i}}\right), t\right)$ are called the patches of $\mathcal{C}=\Gamma([0,1])$. Furthermore, if $P_{l, D_{l}}=P_{0,0}$ we say that the curve $\mathcal{C}$ is closed or that it is a loop.

We denote $\mathcal{B}_{N, D}$ the set of uniform piecewise Bézier curves built from $N$ patches of degree $D$. This clearly a finite dimensional subvariety of $\mathcal{C}^{0}([0,1], E)$ as the image of the following map:

$$
\Psi_{N, D}:\left\{\begin{array}{l}
\left(E^{D+1}\right)^{N+1} \longrightarrow \mathcal{C}^{0}([0,1], E) \\
\left(\boldsymbol{P}_{i}, i=0 \ldots N\right) \longmapsto \Gamma\left(\left(\boldsymbol{P}_{i}, i=0 \ldots N\right), t\right)
\end{array}\right.
$$

Clearly, $\Psi_{N, D}$ is onto from $\left(E^{D+1}\right)^{N+1}$ to $\mathcal{B}_{N, D} \subset \mathcal{C}^{0}([0,1], E)$. It is not very difficult to check that $\Psi_{N, D}$ is almost always one-to-one from $\left(E^{D+1}\right)^{N+1}$ to $\mathcal{B}_{N, D}$. So, $\Psi_{N, D}$ is almost everywhere a diffeomorphism between $\left(E^{D+1}\right)^{N+1}$ and $\mathcal{B}_{N, D} \cdot \mathcal{B}_{N, D}$ is endowed with a manifold structure (even a submanifold structure in $\left.\mathcal{C}^{0}([0,1], E)\right)$.

The density of polynomials in the set of continuous functions implies that for $\operatorname{each} \Phi:[0,1] \longrightarrow E$ continuous there exists $\left(\Gamma_{n}(t)\right)_{n \in \mathbb{N}}$ such that $\lim _{n \rightarrow \infty}\left\|\Phi-\Gamma_{n}\right\|_{2}=$ 0 . So, piecewise Bzier curves are a good approximating set for $\mathcal{C}^{0}([0,1], E)$.

\subsection{Sampling map and retraction to $\Psi_{N, D}$}

Definition 2. Let $t_{0}=0<t_{1}<\ldots<t_{D}=1$, we denote $\boldsymbol{t}=\left(t_{0}, \ldots, t_{D}\right)$ the associated subdivision of $[0,1]$, then we define the sampling map $\mathcal{S}_{\boldsymbol{t}}: \mathcal{B}_{1, D} \longrightarrow$ $E^{D+1}$ by $\mathcal{S}_{\boldsymbol{t}}(\Gamma)=\left(\Gamma\left(t_{0}\right), \ldots, \Gamma\left(t_{D}\right)\right)$. 
Proposition 5. The following diagram is commutative:

$$
\begin{aligned}
& E^{D+1} \stackrel{\Psi_{1, D}}{\longrightarrow} \mathcal{C}^{0}([0,1], E) \\
& \underset{B_{t, D}}{\searrow} \downarrow \mathcal{S}_{\boldsymbol{t}}
\end{aligned}
$$

and $\Psi_{N, D}$ is an invertible linear isomorphism between $\mathcal{B}_{1, D}=\operatorname{Im}\left(\Psi_{1, D}\right)$ and $E^{D+1}$ and its inverse is $\Psi_{N, D}^{-1}=B_{\boldsymbol{t}, D}^{-1} \circ \mathcal{S}_{\boldsymbol{t}}$.

Proof. Let $\Gamma(t)=\sum_{j=1}^{N} \sum_{i=0}^{D} P_{j, i} b_{i, D}(t)$, i.e., $\Gamma=\Psi_{N, D}\left(\left(P_{0,0}, \ldots, P_{0, D}\right), \ldots,\left(P_{N, 0}, \ldots, P_{N, D}\right)\right)$, then clearly $\mathcal{S}_{\boldsymbol{t}}(\Gamma)=$ $B_{\boldsymbol{t}, D} \boldsymbol{P}$ where $\boldsymbol{P}=\left(\begin{array}{c}P_{0,0}^{t} \\ \vdots \\ P_{N, D}^{t}\end{array}\right)$, and so $\mathcal{S}_{\boldsymbol{t}} \circ \Psi_{N, D}(\boldsymbol{P})=B_{\boldsymbol{t}, N}(\boldsymbol{P})$. The remainder of the proposition is a consequence of the fact that $B_{\boldsymbol{t}, N}$ is a linear isomorphism.

Proposition 6. Let $t_{1,0}=0<t_{1,1}<\cdots<t_{1, D}=1 / N=t_{2,0}<t_{2,1}<$ $\cdots<t_{2, D}=2 / N=t_{3,0}<\cdots<t_{N, D}=1$, we denote $\boldsymbol{t}=\left(\boldsymbol{t}_{1}, \ldots, \boldsymbol{t}_{N}\right)$ where $\boldsymbol{t}_{i}=\left(t_{0, i}, \ldots, t_{D, i}\right)$ and we define the sampling map $\mathcal{S}_{\boldsymbol{t}, N}: \mathcal{B}_{N, D} \longrightarrow\left(E^{D}\right)^{N}$ by $\mathcal{S}_{\boldsymbol{t}, N}(\Gamma)=\mathcal{S}_{\boldsymbol{t}_{1}} \times \cdots \times \mathcal{S}_{\boldsymbol{t}_{N}}(\Gamma)=\left(\Gamma\left(t_{1,0}\right), \ldots, \Gamma\left(t_{N, D}\right)\right)$. Then $\mathcal{S}_{\boldsymbol{t}, N}$ is a linear isomorphism between $\mathcal{B}_{N, D}$ and $\left(E^{D+1}\right)^{N+1}$.

Proof. It is a simple consequence of the fact that a Cartesian product of isomophisms is an isomorphism. The inverse map is the Cartesian product of the inverse of the component maps.

Proposition 6 is important since it allows to give $\mathcal{B}_{N, D}$ a vector space structure isomorphic to $\left(E^{D+1}\right)^{N+1}$ (and so, of finite dimension). For instance, it allows to transport distance and between other things in $\mathcal{B}_{N, D}$.

In fact, we focus here on a speciale type of sampling. We consider a sampling where $t_{i, 0}=\frac{i}{N}$ and $t_{i, D}=\frac{i+1}{N}$ and $t_{i, j}=t_{i, 0}+\frac{j}{N D}$. We will call this a regular sampling and we will omit the subscript $\boldsymbol{t}$ when we use these samplings. We use regular samplings to simplify the presentation, but all the results presented in this paper have equivalent statements with general sampling. Let us represent each patch by its control polygon: the matrix of $\mathcal{S}_{t, N}$ is $N$ times the Cartesian production of the map $B_{1, D}$ with itself: $B_{1, D} \times \cdots \times B_{1, D}$. This gives us an easy way to solve the following interpolation problem.

Problem 1. Given $M_{0,0}, \ldots, M_{0, D}, \ldots, M_{N, 0}, \ldots, M_{N, D} \in E$, find $\Gamma \in \mathcal{B}_{N, D}$ such that $\mathcal{S}_{N}(\Gamma)=\left(\begin{array}{c}M_{0,0}^{t} \\ \vdots \\ M_{N, D}^{t}\end{array}\right)$. 
Proposition 7. The solution of problem 1 is given by the image by $\Psi_{N, D}$ of:

$$
\left(\begin{array}{ccc}
B_{1, D}^{-1} & & \\
& \ddots & \\
& & B_{1, D}^{-1}
\end{array}\right)\left(\begin{array}{c}
M_{0,0}^{t} \\
\vdots \\
M_{N, D}^{t}
\end{array}\right) .
$$

Proposition (7) implies that $\chi_{\boldsymbol{t}, D}=B_{t, D}^{-1} \circ \mathcal{S}_{\boldsymbol{t}}: \mathcal{B}_{N, D} \longrightarrow E^{D+1}$ is such that $\Psi_{1, D} \circ \chi_{\boldsymbol{t}, D}=\operatorname{Id}_{E^{D+1}}$. It is easy to extend this result to $\Psi_{N, D}$ using $B_{N, D}=$ $B_{1, D} \times \cdots \times B_{1, D}$ satisfying $B_{N, D}^{-1}=B_{1, D}^{-1} \times \cdots \times B_{1, D}^{-1}$.

This approach allows us to project any element of $\mathcal{C}^{0}([0,1], E)$ on $\mathcal{B}_{N, D}$ using $\mathcal{S}_{N}$. Let $\Lambda \in \mathcal{C}^{0}([0,1], E)$, then denoting $\boldsymbol{M}=\left(\Gamma(0), \Gamma\left(\frac{1}{N D}\right), \ldots, \Gamma\left(\frac{N D-1}{N D}\right), \Gamma(1)\right)^{t}$ we have that $\boldsymbol{P}=\mathcal{S}_{N}^{-1}(\boldsymbol{M}) \in \mathcal{B}_{N, D}$ is such that $\Psi_{N, D}(\boldsymbol{P})=B(\boldsymbol{P}, t)$ coincides with $\Lambda([0,1])$ on at least $(D+1)$ points counted with multiplicities on each patch. This comes from the fact that $\chi_{\boldsymbol{t}, D}$ can be extended to $\mathcal{C}^{0}([0,1], E)$.

Our main claim is that, instead of working directly with $\mathcal{B}_{N, D}$, it is easier to work on the "set of control polygons", namely $E^{D+1}$, using sampling and interpolation that give linear isomorphism between control polygons and sets of sampling points on the curves. In what follows, we will always take this point of view.

\subsection{Tangent space $T \mathcal{B}_{N, D}$ and deformation of curve}

Recall that $\Psi_{N, D}$ defines a linear isomorphism between the "space of control polygons" $\left(E^{D+1}\right)^{N+1}$ and the space of piecewise Bézier curves $\mathcal{B}_{N, D}$. We already saw that for any $\gamma(t) \in \mathcal{B}_{N, D}$ then there exists $\boldsymbol{P} \in\left(E^{D+1}\right)^{N+1}$ such that $\Psi_{N, D}(\boldsymbol{P})=\gamma(t)$ is given by $B_{N, D}^{-1} \circ \mathcal{S}_{N}(\gamma)$. This gives the following proposition:

Proposition 8. Consider the map $T \Psi_{N, D}: T\left(E^{D+1}\right)^{N+1} \longrightarrow T \mathcal{B}_{N, D}$. Then for any $\gamma \in \mathcal{B}_{N, D}$ we have that $T \Psi_{N, D}^{-1}(\gamma): T_{\gamma} \mathcal{B}_{N, D} \longrightarrow T_{\chi_{N, D}(\gamma)}\left(E^{D+1}\right)^{N+1}$ is given by $T \Psi_{N, D}\left(\chi_{N, D}(\gamma)\right)^{-1}(\varepsilon)=B_{N, D}^{-1} \circ \mathcal{S}_{N}(\varepsilon)=\chi_{N, D}(\varepsilon)$ for any $\varepsilon(t) \in$ $T_{\gamma} \mathcal{B}_{N, D}$. Moreover, this is a linear isomorphism.

An element of $\varepsilon(t) \in T_{\gamma} \mathcal{B}_{N, D}$ is called a deformation curve. In fact, this proposition allows to express, given a piecewise Bézier curve and a deformation of its control polygon. This is an essential step proving that manipulating a piecewise Bézier curve, it is enough to manipulate its control polygon. This is the object of the following lemma.

Lemma 2. Let $\boldsymbol{P} \in\left(E^{D+1}\right)^{N+1}, \gamma(t)=\Psi_{N, D}(\boldsymbol{P})=B(\boldsymbol{P}, t) \in \mathcal{B}_{N, D}$ and $\varepsilon(t) \in T_{\gamma} \mathcal{B}_{N, D}$, then:

i. $\varepsilon(t)=\Psi_{N, D}\left(\chi_{N, D}(\varepsilon)\right)$.

ii. $\gamma(t)+\varepsilon(t)=\Psi_{N, D}\left(\boldsymbol{P}+\chi_{N, D}(\varepsilon)\right)$. 
This lemma explain how to lift a deformation from the space of curves to the space of control polygons. The vector space structure of both the space of control polygons $\left(E^{D+1}\right)^{N+1}$ and of piecewise Bézier curves $\mathcal{B}_{N, D}$ allows us to avoid the use of computationally difficult concepts such as the exponential map between manifold and its tangent space for instance. This structure also helps to define a simple notion of distance between two such curves.

\section{Applications to shape optimization}

In this section, we show how the preceding formalism can be exploited in the context of shape optimization. An application to a problem of image segmentation is presented to illustrate our purpose.

\subsection{Shape optimization problem}

A typical shape optimisation problemcan be formulated as follows: given a set of admissible shapes $\mathcal{A}$ and a functional $F: \mathcal{A} \rightarrow \mathbb{R}^{+}$find a shape $\alpha \in \mathcal{A}$ such that for all other shapes $\beta \in \mathcal{A}$, we have $F(\alpha) \leqslant F(\beta)$. Generally, one tries to give to the space of admissible shape a structure of manifold in such a way to be able to compute a "shape gradient" $\nabla F(\beta)$ expressing the evolution of the criterium $F$ with respect to a deformation of the shape $\beta$. This is to say that $\nabla F(\beta)$ associates with every point $M \in \beta$ a deformation vector $\nabla F(\beta)(M) \in T_{M} E$. The computation of such a gradient can require sophisticated computations, since very often, even the computation of the criterium itself requires to solve a system of partial differential equations. Many problems can be expressed as a shape optimization problem. The classical approach to solve this kind of problems is to use $\nabla F(\beta)$, when it is computable, in a gradient method to find a local minimum.

To keep the presentation as simple as possible, we focus on geometric optimisation, i.e. we keep the topology of the shape fixed, in the case where the frontier of the admissible shapes are continuous Jordan curves. But the framework presented here can be extended to topological optimization as it is shown in [5] for a special application on a problem of image segmentation. The case treated here received attention because of its deep links with image segmentation and shape recognition (see $[5,3,2]$ for instance).

We denote $\mathcal{C}_{J}^{0}([0,1], E)$ the set of functions parametrizing a Jordan curve and $\mathcal{B}_{N, D}^{c}=\left\{\gamma \in \mathcal{B}_{N, D} \mid \gamma(t)=\gamma(s)\right.$ with $s \neq t \Leftrightarrow(t=0$ and $s=1)$ or $(t=1$ and $\left.s=0)\right\}$. We have $\mathcal{B}_{N, D}^{c} \subset \mathcal{C}_{J}^{0}([0,1], E)$. We denote:

$$
H_{N, D}=\left\{\left(\left(P_{i, j}, j=0 \ldots D\right), i=0 \ldots N\right) \in\left(E^{D}\right)^{N} \mid P_{0,0}=P_{N, D}\right\}
$$

which is a linear subspace of $\left(E^{D+1}\right)^{N+1}$. We then denote $\Psi_{N, D}^{c}=\left.\Psi_{N, D}\right|_{H_{N, D}}$. As above, $\Psi_{N, D}^{c}$ defines a linear isomorphism between $H_{N, D}$ and $\mathcal{B}_{N, D}^{c}$ using $\left.\mathcal{S}_{N}\right|_{\mathcal{B}_{N, D}^{c}}$ and the same $B_{N, D}$ to define its converse explicitly. 


\subsection{Vector field on $\mathcal{B}_{N, D}$ lifted from the shape gradient}

Let $\nabla F$ be a shape, then for each $\alpha \in \mathcal{C}_{J}^{0}([0,1], E)$ and for any $M \in \alpha([0,1])$, $\nabla F$ associates with $M$ an element $\nabla F(\alpha)(M) \in T_{M} E$. Consider now $\alpha \in \mathcal{B}_{N, D}^{c}$ and $\left(\left(M_{0,0}, \ldots, M_{0, D}\right), \ldots,\left(M_{N, 0}, \ldots, M_{N, D}\right)\right)=\mathcal{S}_{N}(\alpha)$, then $M_{0,0}=M_{N, D}$. We

$$
\mathcal{T}_{N, F}(\alpha)=\left(\left(\nabla F(\alpha)\left(M_{i, j}\right), j=0 \ldots D\right), i=0 \ldots D\right)
$$

This represents the sampling of the deformation of the curve implied by the shape gradient $\nabla F$ to $\alpha$. It is not difficult to see that $\mathcal{T}_{N, F}(\alpha) \in T_{\mathcal{S}_{N}(\alpha)}\left(\left(E^{D}\right)^{N}\right)$.

Theorem 1. The map $B_{N, D}^{-1} \circ \mathcal{T}_{N, F}$ associates with each shape gradient $\nabla F$ a vector field on $H_{N, D}$ which correspond to a vector field $V_{F}$ on $\mathcal{B}_{N, D}^{c}$ through $T \Psi_{N, D}^{c}$.

This theorem allows us to interpret the gradient descent method for shape optimization as a algorithm for integrating a vector field in a finite dimensional space. The gradient descent method corresponds to the Euler method. Clearly, this approach suggests to use a better algorithm for vector field integration.

\subsection{Geometry of the vector field and local extrema of shape cost functional}

Proposition 9. Let $\alpha \in \mathcal{C}_{J}^{0}([0,1], E)$ be such that $\nabla F(\alpha)=\mathbf{0}$, i.e. $\nabla F(\alpha)(M)=$ 0 for all $M \in \alpha([0,1])$ and let $\gamma \in \mathcal{B}_{N, D}$ be such that $\gamma\left(\frac{i}{N D}\right)=\alpha\left(\frac{i}{N D}\right)$ for $i \in\{0, \ldots, N D\}$, i.e. $\gamma=\Psi_{N, D}\left(B_{N, D}^{-1} \circ \mathcal{S}_{N}(\alpha)\right)$. Then $\nabla F(\gamma)\left(\gamma\left(\frac{i}{N D}\right)\right)=0$ for $i \in\{0, \ldots, N D\}$ and $V_{F}\left(B_{N, D}^{-1} \circ \mathcal{S}_{N}(\alpha)\right)=\mathbf{0}$. This is to say that a local extremum of $F$ induces a local extremum of its restriction to $\mathcal{B}_{N, D}$ and that this extremum is "lifted" on a singularity of the vector field $V_{F}$ on $H_{N, D}$.

Proof. The deformation curve of $\gamma$ induced by the gradient of $F$ vanishes at at least $(D+1)(N+1)$ points, but it is a "Bézier curve" of degree $D$, so it is a zero polynomial. So, its control polygon is reduced to the origin and then $B_{N, D}^{-1} \circ \mathcal{S}_{N}(\alpha)$ is a singularity of $V_{F}$.

In fact, the vector field $V_{F}$ is associated with the gradient of the function $F \circ \Psi_{N, D}$. This is an heavy constraint on the vector field. For instance, it is easy to see that $\boldsymbol{P}$ is an attractive singularity of $V_{F}$ if and only if $\Psi_{N, D}(\boldsymbol{P})$ is a local minimum of $\left.F\right|_{H_{N, D}}$.

\subsection{Application to a problem of image segmentation}

In this section, we sketch an application to a problem of image segmentation. It is a problem of omnidirectional vision. Previous methods attacked the problem with some success but does not allowed a full real time treatment. They are all based on snake-like algorithms (see [9]). The gradient used to detect edges is a 
classical one based on a Canny filter and it is combined with a balloon force. The best previously known method is such that propagation of the contour was computed using the fast marching algorithm for level set method. This a typical formulation of image segmentation as a shape optimisation problem. In [6], we use piecewise Bézier curves to compute contour propagation and achieve a very fast segmentation algorithm allowing real time treatment even with sequential algorithms (no use of parallelism or special hardware architecture) on a embedded system.
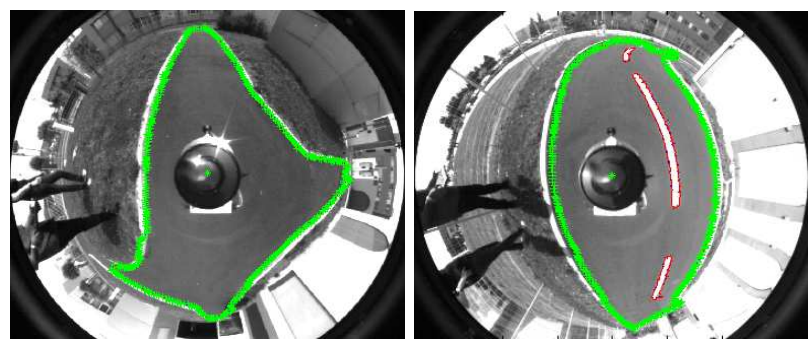

It is very interesting to see that, with a few algorithmic modifications, it is also possible to treat a change of topology, i.e., curves with several connected components as it is shown in the previous figure.

\section{References}

1. P. W. Michor and D. Mumford. An Overview of the Riemannian metric on Spaces of Curves using the Hamiltonian Approach. Applied and Computational Harmonic Analysis, 23 (2007), p. 74-113.

2. A. Yezzi and A. C. G. Mennucci. Metric in space of curves. http://arxiv.org/abs/math/0412454.

3. L. Younes and P. W. Michor and J. Shah and D. Mumford. A metric on shape space with explicit geodesics. Rend. Lincei. Mat. Appl., 9 (2008), p. 25-57.

4. M. Bauer and P. Harms and P. W. Michor. Curvature weighted metrics on shape space of hypersurfaces in $n$-space. Differential Geometry and Applications, 2011.

5. L. Younes. Shapes and Diffeomorphisms. Applied Mathemetical Sciences 171, Springer (2010).

6. O. Labbani-I. and P. Merveilleux-O. and O. Ruatta. Free form based active contours for image segmentation and free space perception, preprint (submitted available to referee at http://www.unilim.fr/pages_perso/olivier.ruatta/TRO-papersubmitted.pdf).

7. A. Henrot and M. Pierre. Variation et optimisation de formes. Mathématiques et Applications, Springer-Verlag Berlin Heidelberg, 2005.

8. J.A. Sethian. Level Set Methods and Fast Marching Methods Evolving Interfaces in Computational Geometry, Fluid Mechanics, Computer Vision, and Materials Science. Cambridge University Press, 1999 Cambridge Monograph on Applied and Computational Mathematics.

9. M. Kass, A. Witkin, and D. Terzopoulos. Snakes: Active contour models. International Journal of Computer Vision (1988), vol. 1, no. 4, pp. 321-331. 